CLINICAL STUDY

\title{
Characterization of resistance to the prolactin-lowering effects of cabergoline in macroprolactinomas: a study in 122 patients
}

\author{
Etienne Delgrange, Tania Daems ${ }^{1}$, Johan Verhelst ${ }^{2}$, Roger $\mathrm{Abs}^{1}$ and Dominique Maiter ${ }^{3}$ \\ Department of Internal Medicine, Cliniques de Mont-Godinne, Mont-Godinne Hospital, Université Catholique de Louvain, B-5530 Mont-sur-Meuse, \\ Belgium, ${ }^{1}$ Department of Endocrinology, University of Antwerp, B-2610 Antwerp, Belgium, ${ }^{2}$ Department of Endocrinology, Middelheim Hospital, \\ B-2610 Antwerp, Belgium and ${ }^{3}$ Department of Endocrinology, Cliniques Saint-Luc, Université Catholique de Louvain, B-1200 Brussels, Belgium \\ (Correspondence should be addressed to E Delgrange; Email: etienne.delgrange@uclouvain.be)
}

\begin{abstract}
Context: Macroprolactinomas poorly responsive to dopamine-agonists are often more aggressive and are usually termed 'resistant' but this clinical concept has always been defined empirically.

Objective: To define resistance to cabergoline $(\mathrm{CAB})$ on the basis of a dose-response relationship established in a large series of macroprolactinoma patients and to assess the influence of gender and tumor invasiveness on the response to treatment.

Design: Retrospective study.

Methods: One hundred and twenty-two patients ( 72 women and 50 men) primarily treated with $\mathrm{CAB}$ for at least 1 year were included. Main outcome measures were serum prolactin (PRL) and tumor size. Results: Normalization of PRL was obtained in 115 out of the 122 patients (94\%). The majority of patients $(96 / 115,83 \%)$ were controlled with a CAB dose $\leq 1.5 \mathrm{mg} /$ week. Most of the other patients $(19 / 26)$ had only a partial resistance, responding to a further increase of the CAB dose. Beyond the dose of $3.5 \mathrm{mg} /$ week, there was no clear advantage in further increasing the dose instead of continuing the treatment at the same dose. Most tumors (98/119 assessable cases, $82 \%)$ showed a significant shrinkage during $\mathrm{CAB}$ treatment. It was more likely to occur in cases of PRL normalization. Both cavernous sinus invasion and male gender were significantly and independently associated with partial or complete resistance to treatment.

Conclusions: Most macroprolactinomas primarily treated with $\mathrm{CAB}$ are adequately controlled with doses $\leq 1.5 \mathrm{mg} /$ week. About $20 \%$ of patients, mainly men and/or those with invasive tumors will require a higher dose of $\mathrm{CAB}$. We suggest defining such patients as resistant to $\mathrm{CAB}$.
\end{abstract}

European Journal of Endocrinology $160747-752$

\section{Introduction}

The sensitivity of prolactinomas to the prolactin (PRL)lowering effects of dopamine agonists (DA) is highly variable. Tumors that are very sensitive to DAs are more likely to show persistent remission of hyperprolactinemia after treatment withdrawal (1), whereas tumors less responsive to medical therapy often express histological markers of cell proliferation (2) and may show a less serendipitous clinical course. These prolactinomas are usually referred to as 'resistant'. However, the concept of resistance to DA therapy has only been empirically defined. In addition, several factors may complicate the analysis of the response of prolactinomas to DA treatment, including diagnostic confusion especially in the case of microprolactinomas (3), spontaneous regression of hyperprolactinemia reported in some patients with microadenomas (4), a poor compliance or intolerance to treatment, more frequently occurring with bromocriptine than cabergoline (CAB) (5), and/or a preceding therapy with a DA possibly modifying the subsequent response to other DAs (6). Recent evidence that chronic CAB treatment, given at high doses in patients with Parkinson's disease, may lead to valvular heart disease $(7,8)$ further reinforces the need to define a dose threshold above which additional PRL suppression is unlikely to occur.

Therefore, the first aim of the present study was to characterize more precisely macroprolactinomas that are poorly responsive or resistant to a prolonged $\mathrm{CAB}$ treatment on the basis of a dose-response relationship established in a large series of cases treated with $\mathrm{CAB}$ for at least 1 year as first-line treatment. A secondary end point was to reassess the influence of gender and of tumor invasiveness on the sensitivity of macroprolactinomas to the PRL-lowering effect of CAB and to look for the median dose able to normalize serum PRL according to these parameters. 


\section{Subjects and methods}

\section{Subjects}

The study was conducted at the departments of endocrinology of the University of Antwerp, the Middelheim Hospital of Antwerp and the University Hospitals Saint-Luc in Brussels and Mont-Godinne in Yvoir, Belgium. All de novo patients presenting between 1992 and 2006 with a pituitary macroadenoma (defined as a tumor of at least $10 \mathrm{~mm}$ in one diameter), a pre-treatment serum PRL concentration $>100 \mu \mathrm{g} / \mathrm{l}$ (2100 mU/l) (9), without biochemical evidence of acromegaly, and who received a primary treatment with $\mathrm{CAB}$ for at least 1 year, at a starting dose between 0.25 and $1.00 \mathrm{mg}$ weekly were considered for inclusion in this retrospective study. Of the 123 patients diagnosed with macroprolactinoma and recruited according to these criteria, one had to be excluded from the analysis because of poor tolerance to $\mathrm{CAB}$ and 122 were finally included ( 72 women and 50 men; aged 13-80 years).

\section{Treatment protocol}

In all but two of the 122 patients, the treatment with CAB had started with a dose of 0.50 or $1.00 \mathrm{mg}$ per week. In the remaining two patients, the initial dose was $0.25 \mathrm{mg}$ weekly but was later increased to at least $0.50 \mathrm{mg}$ weekly. Dose adjustment was carried out on the basis of PRL suppression. In the absence of a significant serum PRL decrease, the weekly dose of $\mathrm{CAB}$ was increased by $0.50 \mathrm{mg}$ every $1-2$ months. In patients not normalizing PRL levels, the maximal $\mathrm{CAB}$ dose was $3.50 \mathrm{mg}$ per week except in four patients in which the dose was further increased. In patients where the PRL levels declined to $<5 \mu \mathrm{g} / \mathrm{l}$, the dose was reduced.

\section{Imaging studies}

Magnetic resonance imaging (MRI) studies were performed in all patients before $\mathrm{CAB}$ treatment. The maximal craniocaudal tumor diameter (height) was calculated on a vertical axis and expressed in $\mathrm{mm}$. Evolution of tumor height was available in all but three patients and was estimated by comparing the initial and the final MRI documents performed at least one year after initiation of CAB therapy. Tumor shrinkage was considered significant when more than 30\% reduction in craniocaudal diameter was observed. On the pre-treatment MRI, the percentage of encasement of the intracavernous segment of the internal carotid artery was recorded and tumors encasing more than three quarters of the artery were considered as invasive (modified from Cottier et al. (10) as previously described (11)).

\section{PRL assays}

Serum PRL was measured with commercially available kits. The upper range of normal was less than $15 \mu \mathrm{g} / \mathrm{l}$ in males and less than $25 \mu \mathrm{g} / \mathrm{l}$ in females. The converting factor was $1 \mu \mathrm{g} / \mathrm{l}=21 \mathrm{mU} / \mathrm{l}$.

\section{Statistical analysis}

Statistical analyses were carried out using SPSS statistical software, version 13.0 (SPSS Inc., Chicago, IL, USA). Data are expressed as means \pm s.E.M. and as medians. Medians were compared using the nonparametric Kruskal-Wallis test, and frequencies using the $\chi^{2}$ test. The independent influence of various parameters (gender, basal PRL level, tumor size, and cavernous sinus invasion) on the sensitivity to the PRL-lowering effect of $\mathrm{CAB}$ was tested by multivariate logistic regression analyses, with stepwise selection of variables by the likelihood-ratio test.

\section{Results}

During the course of $\mathrm{CAB}$ treatment, complete normalization of serum PRL levels was ultimately obtained in 115 of the 122 patients (94\%). The distribution of the weekly dose of CAB necessary to achieve normo prolactinemia is shown in Fig. 1. The median dose in these hormonally controlled patients was $1.0 \mathrm{mg} /$ week, and the majority of them $(82 / 115,71 \%)$ were controlled with either 1.0 or $1.5 \mathrm{mg} /$ week. The proportion of patients responding to doses lower than $1.0 \mathrm{mg} /$ week $(14 / 115,12 \%)$ was probably underestimated because the starting dose was often $1.0 \mathrm{mg}$ weekly. Among the 26 patients who were not controlled with a CAB dose of $1.5 \mathrm{mg} /$ week or lower, nine showed PRL normalization under $2.0-2.5 \mathrm{mg} /$ week and two additional patients responded to a further increase of the $\mathrm{CAB}$ dose to $3.5 \mathrm{mg} /$ week. In the remaining 15 patients, PRL concentration was not normalized after 1 year of treatment, despite a weekly CAB dose

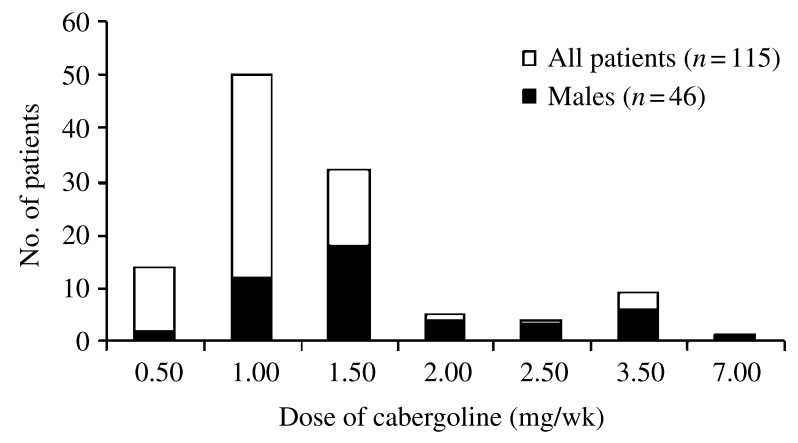

Figure 1 Frequency distribution of the cabergoline weekly doses necessary to obtain complete normalization of serum PRL levels in 115 hormonally controlled macroprolactinomas. 
of $3.5 \mathrm{mg}$. The same dose was maintained in 11 subjects, whereas in four subjects it was progressively increased to $5.25,7.0(n=2)$ and $10.5 \mathrm{mg}$. Among these four patients, PRL normalization was obtained in only one case after 55 months of treatment, with a weekly dose of $7.0 \mathrm{mg}$. However, it could later be reduced to $3.5 \mathrm{mg} /$ week without loss of PRL control. In the 11 patients maintained on a dose of $3.5 \mathrm{mg} /$ week, PRL normalization was finally obtained in seven cases after a mean treatment time of 37 months (15-72 months). Thus, most of the patients (19/26) who did not respond to a $\mathrm{CAB}$ dose $\leq 1.5 \mathrm{mg} /$ week had only partial resistance to the DA, showing PRL normalization after a further increase of the $\mathrm{CAB}$ dose or, more frequently, after a prolonged treatment time. Finally, only seven patients $(6 \%)$ were truly and persistently resistant to medical treatment.

The relationship between the dose of $\mathrm{CAB}$ and the hormonal response was different when comparing noninvasive $(n=101)$ and invasive $(n=21)$ tumors (Fig. 2$)$. Five out of the seven patients with hormonal resistance to $\mathrm{CAB}$ had invasive tumors, so that cavernous sinus invasiveness was associated with more than 10-fold increased risk of resistance to medical treatment (odds ratio 15.5 ; 95\% confidence interval, 2.8-86.7). When considering only the responsive tumors $(n=115)$, the median dose able to normalize PRL level was significantly higher for invasive than for non-invasive tumors ( 1.5 vs $1.0 \mathrm{mg} /$ week; $P<0.001)$. The proportion of patients requiring a $\mathrm{CAB}$ dose $>1.5 \mathrm{mg} /$ week to normalize PRL was also higher when the pituitary tumor was invasive $(7 / 16,44 \%)$ compared to noninvasive macroadenomas $(12 / 99,12 \%$; $P<0.005)$.

Macroprolactinomas in men were more frequently invasive $(15 / 50,30 \%)$ than in women $(6 / 72,8 \%$; $P<0.005)$, and were also less responsive to $\mathrm{CAB}$ (Fig. 1). Thus, 18 out of the 26 patients with partial or complete resistance to $\mathrm{CAB}$ were men (Table 1 ).

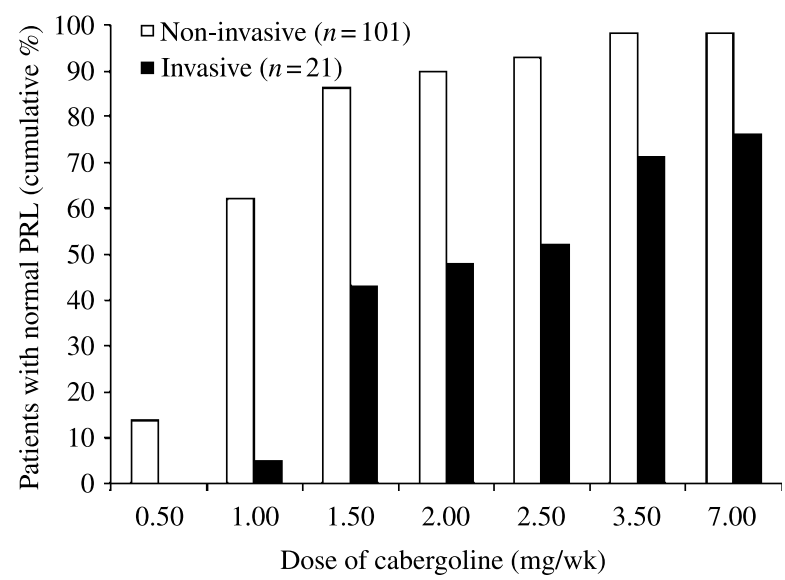

Figure 2 Relationship between the weekly cabergoline dose and the hormonal response in non-invasive $(n=101)$ and invasive $(n=21)$ macroprolactinomas.
Even when considering non-invasive tumors only, the median $\mathrm{CAB}$ dose necessary to obtain PRL normalization was still significantly higher in men than in women ( 1.5 vs $1.0 \mathrm{mg} /$ week; $P<0.001)$. In addition, the proportion of non-invasive tumors showing partial or complete resistance to $\mathrm{CAB}$ also tended to be higher in men $(8 / 35,23 \%)$ than in women $(6 / 66,9 \%$; $P=0.06)$. Half the patients $(63 / 122)$ achieved nadir PRL levels $\leq 5 \mu \mathrm{g} / \mathrm{l}$ with a weekly dose $\leq 1.5 \mathrm{mg}$. These 'highly responsive' tumors were more frequently encountered in women $(45 / 72,63 \%)$ than in men (18/50, 36\%; $P=0.004)$.

When tumors were classified into four groups according to their sensitivity to the PRL lowering effect of $\mathrm{CAB}$ (highly sensitive tumors achieving a nadir PRL level $\leq 5 \mu \mathrm{g} / \mathrm{l}$; responsive tumors showing PRL normalization $>5 \mu \mathrm{g} / \mathrm{l}$ with a $\mathrm{CAB}$ dose $\leq 1.5 \mathrm{mg} /$ week; tumors with partial resistance; tumors with complete resistance), both tumor invasiveness and male gender were significantly and independently associated with partial or complete resistance to $\mathrm{CAB}$, while this association was only significant for invasiveness when considering the seven patients with complete resistance (Tables 1 and 2).

Most tumors (98/119 assessable cases, 82\%) showed a significant shrinkage during $\mathrm{CAB}$ therapy. Neither tumor invasiveness nor gender was predictive of tumor shrinkage but it was more likely to occur in cases of PRL normalization (95/112 vs 3/7 in cases of hormonal resistance; $P=0.005)$. A pronounced tumoral regression (residual tumor height $\leq 5 \mathrm{~mm}$ ) was never observed in resistant tumors (0/7) and in only 2 out of 19 tumors partially resistant to $\mathrm{CAB}$, but was otherwise obtained in 34 of 93 responders $(37 \%, P=0.006)$.

\section{Discussion}

Our study confirms that $\mathrm{CAB}$ given as first-line treatment in macroprolactinomas is highly effective in achieving both PRL normalization and tumor shrinkage $(12,13)$. Indeed, hormonal and tumoral responses were obtained in 94 and $82 \%$ of the cases respectively. This is in keeping with several previous studies (14-18), and an overview of the reported numbers of patients with previously untreated macroprolactinoma responding to long-term treatment with $\mathrm{CAB}$ is shown in Table 3.

In the absence of PRL normalization and/or tumor shrinkage, current recommendation is to progressively increase the dose of $\mathrm{CAB}(12,13,19)$, as no major side effects of long-term treatment have been reported yet in the setting of hyperprolactinemia. Furthermore, in the absence of established doses at which $\mathrm{CAB}$ has its maximal antisecretory and/or antitumoral effects, very high doses have been used occasionally (up to 1.0$3.0 \mathrm{mg}$ daily). This attitude seems today inadvisable with the recent recognition that chronic CAB therapy may induce valvular dysfunction in patients with 
Table 1 Characteristics of prolactinomas according to their sensitivity to the prolactin (PRL)-lowering effect of cabergoline.

\begin{tabular}{lllllr}
\hline & $\begin{array}{l}\text { Highly sensitive } \\
\text { tumors }\end{array}$ & $\begin{array}{l}\text { Responsive } \\
\text { tumors }\end{array}$ & $\begin{array}{l}\text { Partial } \\
\text { resistance }\end{array}$ & $\begin{array}{l}\text { Complete } \\
\text { resistance }\end{array}$ \\
\hline$N$ & 63 & 33 & 19 & 7 & \\
Male sex, $n(\%)$ & $18(29)$ & $14(42)$ & $14(74)$ & $4(57)$ & 0.0042 \\
Basal PRL level $(\mu \mathrm{g} / \mathrm{l})$ & $736 \pm 130(314)$ & $974 \pm 406(443)$ & $2816 \pm 976(1293)$ & $8387 \pm 5735(1203)$ & 0.0015 \\
Tumor height $(\mathrm{mm})$ & $17 \pm 1(15)$ & $18 \pm 1(16)$ & $24 \pm 3(18)$ & $33 \pm 9(26)$ & 0.0707 \\
Giant prolactinomas, $n(\%)$ & $3(5)$ & $1(3)$ & $3(16)$ & $2(29)$ & 0.0044 \\
Cavernous sinus invasion, $n(\%)$ & $6(10)$ & $3(9)$ & $7(37)$ & $5(71)$ & 0.0001 \\
Tumor shrinkage, $n(\%)$ & $55 / 62(89)$ & $25 / 31(81)$ & $15 / 19(79)$ & $3 / 7(43)$ & 0.0239 \\
Residual tumor $\leq 5 \mathrm{~mm}, n(\%)$ & $21 / 62(34)$ & $13 / 31(42)$ & $2 / 19(11)$ & $0 / 7(0)$ & 0.0302 \\
Time to PRL normalization, & $3 \pm 0(2)$ & $12 \pm 5(4)$ & $23 \pm 5(17)$ & $44 \pm 15(45)$ &
\end{tabular}

Tumors were classified into four groups according to their sensitivity to the PRL lowering effect of cabergoline: highly sensitive tumors achieving a nadir PRL level $\leq 5 \mu \mathrm{g} / \mathrm{l}$; responsive tumors showing $\mathrm{PRL}$ normalization (but $>5 \mu \mathrm{g} / \mathrm{l}$ ) with a weekly cabergoline dose $\leq 1.5 \mathrm{mg}$; tumors with partial resistance requiring a cabergoline dose $\geq 2.0 \mathrm{mg} /$ week to normalize PRL; complete resistance in the absence of normalization of PRL despite increasing the weekly dose of cabergoline to $3.5 \mathrm{mg}$ for at least 1 year. Data are shown as mean \pm S.E.M. (median) or prevalence as number and percentage in parentheses. $P$ values were calculated using the non-parametric Kruskal-Wallis and $\chi^{2}$ tests.

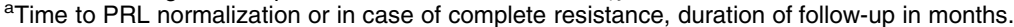

Parkinson's disease $(7,8)$. Indeed, the drug is also a serotonin $2 \mathrm{~B}$ receptor agonist and activation of this receptor is a key step in initiating drug-induced valvular heart disease (8). Moreover, in such conditions, the cardiac side effects seem to be related to the cumulative exposure to the DA $(20,21)$. Therefore, although a few retrospective echocardiographic studies recently published in patients with prolactinomas chronically treated with $\mathrm{CAB}$ are reassuring and show no close relationship between the cumulative dose of the DA and the presence of valve regurgitation (review in (22)), it should be recommended to use the lowest effective dose of $\mathrm{CAB}$ and to define a dose threshold above which further response is unlikely to occur.

There is no widely accepted or evidence-based definition of the concept of resistance to DA therapy $(12,13,19)$. In former studies, resistance to bromocriptine was generally defined as an absence of normalization of PRL levels despite increasing the daily dose up to $15 \mathrm{mg}$ for at least 3 months. Using this definition, bromocriptine-resistant tumors have been shown to exhibit higher proliferative activity (2) and a more severe clinical course (23). The dose of $15 \mathrm{mg} /$ day appears to be twofold higher than the average dose able to control hyperprolactinemia in patients with macroprolactinoma (17). For CAB, using similar criteria, a dose of $2.0 \mathrm{mg}$ per week was proposed to define resistance to treatment in macroprolactinomas (17). Later on, it was proposed to distinguish two definitions of resistance: failure to normalize PRL levels and failure to reduce tumor size. However, there are obvious drawbacks in using failure to reduce tumor size for adjusting treatment doses and defining resistance to treatment: the absence of uniform criteria to appreciate tumor size reduction, the absence of established doseresponse relationship regarding tumor shrinkage and, in patients showing normalization of PRL levels without change in tumor size, the possibility of an alternative histological diagnosis. Thus, even if the desired biological response in the treatment of hyperprolactinemia is to restore gonadal function rather than normalizing PRL levels, the latter is an easily assessable endpoint which has demonstrated its clinical relevance and the lack of PRL normalization remains the mainstay in defining resistance to treatment. Furthermore, normo prolactinemia is usually required to obtain complete tumoral response and thus to consider treatment withdrawal (12).

In most macroprolactinomas primarily treated with $\mathrm{CAB}$, normo prolactinemia can be achieved with reasonably low doses. In the present study, about $80 \%$ of the patients respond to doses ranging from 0.5 to $1.5 \mathrm{mg} /$ week. Above this dose, each increment will result in PRL normalization in only a few patients and

Table 2 Factors predicting partial or complete resistance to cabergoline according to univariate or multivariate analyses.

\begin{tabular}{|c|c|c|c|c|c|c|}
\hline \multirow[b]{2}{*}{ Variable } & \multicolumn{3}{|c|}{ Univariate } & \multicolumn{3}{|c|}{ Multivariate } \\
\hline & Odds ratio & $95 \% \mathrm{Cl}$ & $P$ & Odds ratio & $95 \% \mathrm{Cl}$ & $P$ \\
\hline Male gender & 4.500 & $1.767-11.459$ & 0.002 & 3.891 & $1.236-12.248$ & 0.020 \\
\hline Basal PRL ${ }^{a}$ & 1.001 & $1.000-1.002$ & 0.007 & 1.000 & $1.000-1.000$ & NS \\
\hline Tumor height ${ }^{a}$ & 1.068 & $1.026-1.113$ & 0.002 & 0.933 & $0.850-1.025$ & NS \\
\hline Invasiveness & 8.286 & 2.951-23.261 & $<0.001$ & 11.744 & $1.805-76.416$ & 0.010 \\
\hline
\end{tabular}

$\mathrm{Cl}$, confidence interval.

${ }^{a}$ Odds ratio are given for each additional unit. 
Table 3 Overview of the response to long-term ( $\geq 1$ year) treatment with cabergoline in previously untreated macroprolactinomas.

\begin{tabular}{lllll}
\hline $\begin{array}{l}\text { Author, date and } \\
\text { reference }\end{array}$ & $\begin{array}{l}\text { Number of patients } \\
\text { (females/total) }\end{array}$ & $\begin{array}{l}\text { PRL normalization, } \\
\boldsymbol{n}(\%)\end{array}$ & $\begin{array}{l}\text { Tumor } \\
\text { shrinkage, } \boldsymbol{n}(\%)\end{array}$ & $\begin{array}{l}\text { Tumor } \\
\text { disappearance, } \boldsymbol{n}(\%)\end{array}$ \\
\hline Cannavò et al. (1999) (14) & $10 / 11$ & $11 / 11(100)$ & ND & $4 / 11(36)$ \\
Pontikides et al. (2000) (15) & $6 / 12$ & $12 / 12(100)$ & $8 / 12(67)$ & $0 / 12(0)$ \\
George et al. (2000) (16) & $2 / 8$ & $7 / 8(88)$ & $3 / 8(38)$ & $0 / 8(0)$ \\
Di Sarno et al. (2001) (17) & $29 / 56$ & $46 / 56(82)$ & $47 / 56(84)$ & $8 / 56(14)$ \\
Colao et al. (2004) (18) & $0 / 41$ & $31 / 41(76)$ & $41 / 41(100)$ & $15 / 41(37)$ \\
Present study & $72 / 122$ & $115 / 122(94)$ & $98 / 119(82)$ & $5 / 122(4)$ \\
Total & $119 / 250$ & $222 / 250(89)$ & $197 / 236(83)$ & $32 / 250(13)$ \\
\hline
\end{tabular}

this might also be related to the prolonged treatment time. Thus, we suggest to call the patients requiring a dose of $\mathrm{CAB}>1.5 \mathrm{mg} /$ week resistant to treatment. Although this resistance to the PRL-lowering effect of $\mathrm{CAB}$ is often partial and can be overcome in $75 \%$ of the cases by increasing the weekly dose up to $3.5 \mathrm{mg}$, we would suggest considering neurosurgery as an alternative (24). However, if the medical treatment option is maintained, there is no outspoken advantage in further increasing the dose above $3.5 \mathrm{mg} /$ week.

We also reassessed the influence of cavernous sinus invasion and of male gender on the dose of CAB needed to normalize PRL. When cavernous sinus invasion is present, normo prolactinemia is obtained with $\mathrm{CAB}$ doses of $\leq 1.5 \mathrm{mg} /$ week in a minority of cases $(43 \%$ in our study) and complete tumoral response is unlikely to occur, as only 2 of the 21 invasive tumors showed major shrinkage. Prolactinomas in men are less sensitive to DA therapy than in women. We have previously shown that resistance to the PRL-lowering effect of bromocriptine was more frequent among males with a prevalence of $30 \%$ compared to $5 \%$ in females (25). In one large study using $\mathrm{CAB}$, this figure was still 25\% (26) and in another study using $\mathrm{CAB}$ as first-line treatment, the resistance rate in males was $24 \%$ (18) compared to no more than $10 \%$ in females. It has also been shown that DA resistance increases the risk of non-surgical (spontaneous or DA induced) cerebrospinal fluid rhinorrhea in patients with an invasive macroprolactinoma and that this complication occurs mainly in men (27). We demonstrate here that in patients with a non-invasive macroprolactinoma responding to $\mathrm{CAB}$, the dose necessary to obtain PRL normalization is still significantly higher in males than in females. Finally, when $\mathrm{CAB}$ is withdrawn after long-term treatment, the recurrence rate of hyperprolactinemia is higher in males than in females (1).

In conclusion, $\mathrm{CAB}$ is effective at doses ranging from 0.5 to $1.5 \mathrm{mg}$ weekly in most macroprolactinomas. However, about $20 \%$ of patients, mainly men and/or patients with invasive tumors will require a higher dose and should be defined as resistant to treatment. These patients are also less likely to show a significant tumoral response during treatment. Further studies are needed to assess the rate of remission of hyperprolactinemia after treatment withdrawal in this subset of patients and the risk of fibrotic valvular heart disease in case of chronic treatment.

\section{Declaration of interest}

The authors declare that there is no conflict of interest that could be perceived as prejudicing the impartiality of the research reported.

\section{Funding}

This research did not receive any specific grant from any funding agency in the public, commercial or not-for-profit sector.

\section{References}

1 Colao A, Di Sarno A, Guerra E, Pivonello R, Cappabianca P, Caranci F, Elefante A, Cavallo LM, Briganti F, Cirillo S \& Lombardi G. Predictors of remission of hyperprolactinaemia after long-term withdrawal of cabergoline therapy. Clinical Endocrinology 200767 426-433.

2 Delgrange E, Sassolas G, Perrin G, Jan M \& Trouillas J. Clinical and histological correlations in prolactinomas, with special reference to bromocriptine resistance. Acta Neurochirurgica 2005147 $751-758$.

3 Feigenbaum SL, Downey DE, Wilson CB \& Jaffe RB. Transsphenoidal pituitary resection for preoperative diagnosis of prolactinsecreting pituitary adenoma in women: long term follow-up. Journal of Clinical Endocrinology and Metabolism $1996 \mathbf{8 1}$ 1711-1719.

4 Schlechte J, Dolan K, Sherman B, Chapler F \& Luciano A. The natural history of untreated hyperprolactinemia: a prospective analysis. Journal of Clinical Endocrinology and Metabolism 198968 412-418.

5 Webster J, Piscitelli G, Polli A, Ferrari CI, Ismail I \& Scanlon MF for the Cabergoline Comparative Study Group A comparison of cabergoline and bromocriptine in the treatment of hyperprolactinemic amenorrhea. New England Journal of Medicine 1994331 904-909.

6 Colao A, Di Sarno A, Landi ML, Scavuzzo F, Cappabianca P, Pivonello R, Volpe R, Di Salle F, Cirillo S, Annunziato L \& Lombardi G. Macroprolactinoma shrinkage during cabergoline treatment is greater in naive patients than in patients pretreated with other dopamine agonists: a prospective study in 110 patients. Journal of Clinical Endocrinology and Metabolism $2000 \mathbf{8 5}$ $2247-2252$

7 Pinero A, Marcos-Alberca P \& Fortes J. Cabergoline-related severe restrictive mitral regurgitation. New England Journal of Medicine $20053531976-1977$.

8 Roth BL. Drugs and valvular heart disease. New England Journal of Medicine 2007356 6-9.

9 Karavitaki N, Thanabalasingham G, Shore HCA, Trifanescu R, Ansorge O, Meston N, Turner HE \& Wass JAH. Do the limits of serum prolactin in disconnection hyperprolactinaemia need 
re-definition? A study of 226 patients with histologically verified non-functioning pituitary macroadenoma Clinical Endocrinology 200665 524-529.

10 Cottier JP, Destrieux C, Brunereau L, Bertrand P, Moreau L, Jan M \& Herbreteau D. Cavernous sinus invasion by pituitary adenoma: MR imaging. Radiology 2000215 463-469.

11 Delgrange E, Duprez T \& Maiter D. Influence of parasellar extension of macroprolactinomas defined by magnetic resonance imaging on their responsiveness to dopamine agonist therapy. Clinical Endocrinology $2006 \mathbf{6 4} 456-462$.

12 Gillam MP, Molitch ME, Lombardi G \& Colao A. Advances in the treatment of prolactinomas. Endocrine Reviews 200627 485-534.

13 Olafsdottir A \& Schlechte J. Management of resistant prolactinomas. Nature Clinical Practice. Endocrinology \& Metabolism 20062 552-561.

14 Cannavò S, Curtò L, Squadrito S, Almoto B, Vieni A \& Trimarchi F. Cabergoline: a first-choice treatment in patients with previously untreated prolactin-secreting pituitary adenoma. Journal of Endocrinological Investigation 199922 354-359.

15 Pontikides N, Krassas GE, Nikopoulou E \& Kaltsas T. Cabergoline as a first-line treatment in newly diagnosed macroprolactinomas. Pituitary 20002 277-281.

16 George LD, Nicolau N, Scanlon MF \& Davies JS. Recovery of growth hormone secretion following cabergoline treatment of macroprolactinomas. Clinical Endocrinology 200053 595-599.

17 Di Sarno A, Landi ML, Cappabianca P, Di Salle F, Rossi FW, Pivonello R, Di Somma C, Faggiano A, Lombardi G \& Colao A. Resistance to cabergoline as compared with bromocriptine in hyperprolactinemia: prevalence, clinical definition, and therapeutic strategy. Journal of Clinical Endocrinology and Metabolism $2001865256-5261$.

18 Colao A, Vitale G, Cappabianca P, Briganti F, Ciccarelli A, De Rosa M, Zarrilli S \& Lombardi G. Outcome of cabergoline treatment in men with prolactinoma: effects of a 24-month treatment on prolactin levels, tumor mass, recovery of pituitary function, and semen analysis. Journal of Clinical Endocrinology and Metabolism $2004891704-1711$.

19 Molitch ME. Pharmacologic resistance in prolactinomas patients. Pituitary 20058 43-52.
20 Zanettini R, Antonini A, Gatto G, Gentile R, Tesei S \& Pezzoli G. Valvular heart disease and the use of dopamine agonists for Parkinson's disease. New England Journal of Medicine 2007356 39-46.

21 Schade R, Andersohn F, Suissa S, Haverkamp W \& Garbe E. Dopamine agonists and the risk of cardiac-valve regurgitation. New England Journal of Medicine 2007356 29-38.

22 Kars M, Pereira AM, Bax JJ \& Romijn JA. Cabergoline and cardiac valve disease in prolactinoma patients: additional studies during long-term treatment are required. European Journal of Endocrinology 2008159 363-367.

23 Brue T, Pellegrini I, Priou A, Morange I \& Jaquet P. Prolactinomas and resistance to dopamine agonists. Hormone Research 199238 84-89.

24 Hamilton DK, Vance ML, Boulos PT \& Laws ER. Surgical outcomes in hyporesponsive prolactinomas: analysis of patients with resistance or intolerance to dopamine agonists. Pituitary 20058 53-60.

25 Delgrange E, Trouillas J, Maiter D, Donckier J \& Tourniaire J. Sexrelated difference in the growth of prolactinomas: a clinical and proliferation markers study. Journal of Clinical Endocrinology and Metabolism 199782 2102-2107.

26 Verhelst J, Abs R, Maiter D, Van den Bruel A, Vandeweghe M, Velkeniers B, Mockel J, Lamberigts G, Petrossians P, Coremans P, Mahler C, Stevenaert A, Verlooy J, Raftopoulos C \& Beckers A. Cabergoline in the treatment of hyperprolactinemia: a study in 455 patients. Journal of Clinical Endocrinology and Metabolism 1999 $842518-2522$.

27 Suliman SGI, Gurlek A, Byrne JV, Sullivan N, Thanabalasingham G, Cudlip S, Ansorge O \& Wass JAH. Nonsurgical cerebrospinal fluid rhinorrhea in invasive macroprolactinoma: incidence, radiological, and clinicopathological features. Journal of Clinical Endocrinology and Metabolism 2007 92 3829-3835.

Received 4 February 2009

Accepted 13 February 2009 